\title{
Metabolic Syndrome, Wall Motion Score and Infarct Size after AMI
}

\section{Leonardo S. Roever-Borges* and Resende ES}

Department of Clinical Research, Federal University of Uberlândia, Brazil

*Corresponding author: Leonardo S. Roever-Borges, Av. Para, 1720 - Bairro Umuarama, Uberlândia - MG - CEP 38400-902, Brazil, Tel: +553488039878; E-mail: leonardoroever@hotmail.com

Received date: August 03, 2015; Accepted date: August 07, 2015; Published date: August 10, 2015

Copyright: ( 2015 Roever L. This is an open-access article distributed under the terms of the Creative Commons Attribution License, which permits unrestricted use, distribution, and reproduction in any medium, provided the original author and source are credited.

\section{Editorial}

The infarct size is an important marker for early and late mortality after acute myocardial infarction [1,2]. The metabolic syndrome (MetS) is interrelated atherosclerotic risk factors including insulin resistance, hypertension, a low level of high-density lipoprotein (HDL) cholesterol, a high triglyceride level, a high plasma glucose concentration, central obesity, and the increased number of components of the metabolic syndrome is associated with a higher mean carotid intima media thickness, lower ankle brachial pressure index in patients with coronary heart disease, peripheral arterial disease, or abdominal aortic aneurysm [3-5].

Hajsadeghi et al. reported a study with 100 patients with MetS (group I) and 100 control (group II) who experienced acute myocardial infarction. In this study the left ventricle (LV) was divided into six basal segments (anterior, anterolateral, inferolateral, inferior, inferoseptal, and anteroseptal), six middle segments (same subgroups), and four apically located segments (anterior, septal, inferior, and posterior). By visual analysis of systolic wall thickening, segments were assigned a wall motion score (WMS) as follows:

- Normal or hyperkinetic (normal endocardial excursion and systolic wall thickening);

- Hypokinetic (reduced excursion and wall thickening);

- Akinetic (absent excursion and wall thickening); and

- Dyskinetic (paradoxical systolic outward wall motion).

WMS index (WMSI) was calculated by dividing the sum of all WMS by the total number of segments analyzed [1].

The authors found that the peak CK-MB and cTnI in 72 hours it was significantly higher in patients with MetS compared with control subjects $(\mathrm{P}<0.001)$. After 72 hours patients with metabolic syndrome have markedly superior wall motion abnormality assessed by echocardiography derived wall motion score index (WMSI) (P $<0.001)$. Also found statistically significant relationships between WMSI and peak CK-MB and also cTnI at 72 hours $(\mathrm{P}<0.001)(1)$.

Logstrup et al. studied the effects of known DM, newly diagnosed $\mathrm{DM}$, and impaired glucose tolerance (IGT) on echocardiographyderived coronary flow reserve (CFR) in a group of patients with recent AMI. The authors an association between a decreased CFR and the diagnosed DM but not the IGT [6].

The patients with MetS have significantly higher infarct size compared to those without metabolic syndrome.

\section{References}

1. Hajsadegh S, Chitsazan M, Chitsazan M, Haghjoo M, Babaali N Norouzzadeh Z,et. al. (2015) Metabolic Syndrome is Associated With Higher Wall Motion Score and Larger Infarct Size After Acute Myocardial Infarction. Res Cardiovasc Med 4: e25018.

2. Miller TD, Christian TF, Hopfenspirger MR, Hodge DO, Gersh BJ, et al. (1995) Infarct size after acute myocardial infarction measured by quantitative tomographic $99 \mathrm{mTc}$ sestamibi imaging predicts subsequent mortality. Circulation 92: 334-341.

3. Wilson PW, Kannel WB, Silbershatz H, D'Agostino RB (1999) Clustering of metabolic factors and coronary heart disease. Arch Intern Med 159: 1104-1109.

4. Grundy SM (1999) Hypertriglyceridemia, insulin resistance, and the metabolic syndrome. Am J Cardiol 83: 25f-9f.

5. Olijhoek JK, van der Graaf Y, Banga JD, Algra A, Rabelink TJ, et al (2004) The metabolic syndrome is associated with advanced vascular damage in patients with coronary heart disease, stroke, peripheral arterial disease or abdominal aortic aneurysm. Eur Heart J 25: 342-348.

6. Logstrup BB, Hofsten DE, Christophersen TB, Moller JE, et al. (2009) Influence of abnormal glucose metabolism on coronary microvascular function after a recent myocardial infarction. JACC Cardiovasc Imaging 2: $1159-1166$. 